\title{
Efficacy of Using High-Dose Vitamin C, Thiamine, and Hydrocortisone in Sepsis and Septic Shock: A Retrospective Before-After Study
}

Haytham Wali ( $\sim$ haytham.a.wali@gmail.com )

Columbus Regional Healthcare System https://orcid.org/0000-0001-6279-3809

William Malik

Carondelet St. Joseph's Hospital

Juliane Mayette

Carondelet St. Joseph's Hospital

\section{Research Article}

Keywords: Sepsis, septic shock, vitamin C, ascorbic acid, thiamine, hydrocortisone

Posted Date: July 14th, 2021

DOl: https://doi.org/10.21203/rs.3.rs-487165/v1

License: (c) (i) This work is licensed under a Creative Commons Attribution 4.0 International License.

Read Full License 


\section{Abstract}

Background/Objective Sepsis can be associated with increased production of reactive oxygen species that deplete antioxidant molecules and increase the consumption of vitamin C, which correlates with multiorgan failure and death. Intravenous vitamin C may protect several microvascular functions, including capillary blood flow, microvascular permeability barrier, and arteriolar responsiveness to vasoconstrictors and vasodilators. This study was conducted to assess the practice of administering high-dose vitamin $\mathrm{C}$ in critically ill patients with sepsis or septic shock at our institution retrospectively.

Methods We conducted an electronic health record (EHR)-based, retrospective, before-after study. The primary objective was to assess the efficacy of using a high-dose vitamin $C$ regimen in decreasing hospital mortality. A two-sided P-value of $<0.05$ was considered statistically significant.

Results A total of 84 patient records were included in this study. Administration of high-dose vitamin C, thiamine, and hydrocortisone was associated with higher hospital mortality $(64.3 \%$ versus $42.9 \%$; $=$ 0.049), higher ICU mortality ( $59.5 \%$ versus $42.9 \% ; P=0.07)$, shorter ICU length of stay (three versus seven days; $P=0.53)$, higher incidence of acute kidney injury $(61.9 \%$ versus $26.2 \% ; P=0.001)$, and a higher requirement for renal replacement therapy $(76.9 \%$ versus $45.5 \% ; P=0.06)$.

Conclusion Administration of high-dose vitamin C, thiamine, and hydrocortisone in critical care patients with sepsis or septic shock was associated with higher hospital mortality and higher incidence of acute kidney injury.

\section{Introduction}

Sepsis is an inflammatory syndrome with life-threatening organ dysfunction resulting from a dysregulated host response to infection [1, 2]. According to the United States Centers for Disease Control and Prevention (CDC), at least 1.7 million adults in the United States develop sepsis each year. Also, nearly 270,000 people die as a result of sepsis. Furthermore, one out of three death occurrences in hospitals is associated with sepsis [3]. In a retrospective database analysis of 2,466,605 sepsis cases treated as inpatients between January 2010 and September 2016, sepsis, severe sepsis, and septic shock were associated with $6 \%, 15 \%$, and $34 \%$ mortality rates and costs of approximately $\$ 16,000, \$ 25,000$, and $\$ 38,000$, respectively [4].

Based on the 2016 Surviving Sepsis Campaign International Guidelines for Management of Sepsis and Septic Shock, fundamental management strategies for sepsis or septic shock include early aggressive fluid resuscitation, appropriate early antibiotics, hemodynamic support with vasopressors, and the identification and control of infected sites [5]. The guidelines grade the use of hydrocortisone as a weak recommendation in septic shock unresponsive to fluid resuscitation and vasopressor therapy. Antioxidant supplementation is not part of current sepsis therapy recommendations. 
Sepsis can be associated with increased production of reactive oxygen species (ROS) that deplete antioxidant molecules and increase consumption of vitamin $\mathrm{C}$, which correlates with multiorgan failure and death. Intravenous vitamin $\mathrm{C}$ may protect several microvascular functions, including capillary blood flow, microvascular permeability barrier, and arteriolar responsiveness to vasoconstrictors and vasodilators [6]. High-dose intravenous (IV) vitamin $\mathrm{C}$ has recently been explored as adjunctive therapy in sepsis because of its anti-inflammatory and antioxidant properties [7, 8].

Data have shown conflicting results on the efficacy of using the combination of high-dose vitamin C, thiamine, and hydrocortisone in critically ill patients with sepsis and septic shock. In a retrospective before-after study including 94 patients (47 patients in the treatment group and 47 patients in the control group) with a primary diagnosis of severe sepsis or septic shock, the combination of intravenous vitamin $\mathrm{C}$, moderate-dose hydrocortisone, and thiamine was associated with a significant decrease in-hospital mortality compared with the control group $(8.5 \%$ versus $40.4 \%$, respectively; $\mathrm{P}<0.001)$. Also, the time to wean off vasopressors was significantly decreased in the treatment group $(18.3 \pm 9.8$ hours versus $54.9 \pm$ 28.4 hours in the control group; $\mathrm{P}<0.001$ ) [7].

However, another retrospective cohort study with a similar study design, in an attempt to replicate the results of the above study by Marik and colleagues, found no significant difference in-hospital mortality between the study groups ( $40.4 \%$ in the treatment group versus $40.4 \%$ in the control group; $P=1.000$ ). There was no significant difference in the secondary outcomes as well, including intensive care unit (ICU) mortality, the requirement for renal replacement therapy for acute kidney injury, ICU length of stay, hospital length of stay, and time to vasopressor independence [8].

Using high-dose Vitamin $\mathrm{C}$ alone did not significantly reduce hospital mortality in a retrospective cohort study ( $46 \%$ in the Vitamin $C$ group versus $40 \%$ in the control group; $P=0.62$ ). Furthermore, no significant difference was found in the secondary outcomes, including ICU mortality, 90-day mortality, time to shock reversal, doses of vasopressors used during the first four ICU days, duration of initial mechanical ventilation, changes in Sepsis-related Organ Failure Assessment (SOFA) scores and PaO2/FiO2 ratio during the first four ICU days, use of renal replacement therapy (RRT) in patients with acute kidney injury (AKI), and ICU and hospital lengths of stay [9]. It has been proposed that the combination of vitamin C, thiamine, and hydrocortisone has a synergistic effect on reversing the pathophysiologic changes of sepsis, and it cannot be achieved with each drug alone [7].

Carondelet St. Joseph's (CSJ) hospital is a 486-bed acute-care hospital established in 1961 in Tucson, Arizona, United States. The hospital has 43 adult ICU beds (23 Medical ICU, 8 Surgical/Trauma ICU, and 12 Neuro ICU). The average ICU patient volume is 23 per day. The critical care service at CSJ hospital is provided through Sound Physicians ${ }^{\mathrm{TM}}$, a physician-led healthcare organization based in Tacoma, Washington, United States.

The practice of administering high-dose vitamin C regimen (intravenous vitamin C $1500 \mathrm{mg}$ every six hours; intravenous hydrocortisone $50 \mathrm{mg}$ every six hours; and intravenous thiamine $200 \mathrm{mg}$ every 12 hours) for four days in critically ill patients with sepsis or septic shock was adopted at Carondelet St. 
Joseph's hospital in October 2017. The regimen was developed as an order set in the computerized physician order entry (CPOE). We conducted this study to assess the outcomes of this practice at our institution retrospectively.

\section{Methods}

We conducted an electronic health record (EHR)-based, retrospective, before-after study. Patients were selected by querying the electronic health records (EHRs) and auditing orders for the use of intravenous Vitamin C through Cerner Millennium ${ }^{\circledR}$ (Cerner Corporation, North Kansas City, Missouri, United States) to identify the patients who received the combination of high-dose Vitamin C, thiamine, and hydrocortisone.

Data were collected from October 2017 until September 2019 to account for the treatment group (After). Then, data from a matching number of patients with the same inclusion criteria from October 2015 to September 2017 were collected to account for the control group (Before). Study data were collected and managed using the Research Electronic Data Capture (REDCap ${ }^{\circledR}$ ) version 9.3.3 (Vanderbilt University, Nashville, Tennessee, United States) hosted at The University of Arizona Center for Biomedical Informatics and Biostatistics (CB2) [10, 11].

Patients were included if they were 18 years of age or older; admitted to the intensive care unit with a primary diagnosis of sepsis or septic shock based on the International Classification of Diseases, Tenth Revision (ICD-10); required vasopressors for hypotension management; and required mechanical ventilation. Pregnant women and patients with limitations of care (e.g., comfort care) were excluded.

One of the most significant difficulties with this type of study design is identifying a historical control group accurately. Since it would be challenging to control all the parameters that were measured between the two groups, the Acute Physiology and Chronic Health Evaluation (APACHE II) score, which could help including patients with a similar estimated in-hospital mortality, was used to provide some kind of comparability between the included groups.

The primary endpoint of this study was hospital mortality. Secondary endpoints included ICU mortality, ICU length of stay, and duration of vasopressors. Safety endpoints included the incidence of acute kidney injury (AKI), which was based on the definition of Kidney Disease: Improving Global Outcomes (KDIGO): an increase in serum creatinine ( $\mathrm{SCr}$ ) by $>0.3 \mathrm{mg} / \mathrm{dL}$ within 48 hours; an increase in SCr to $>1.5$ times baseline, which is known or presumed to have occurred within the previous seven days; or urine volume< $0.5 \mathrm{ml} / \mathrm{kg} / \mathrm{h}$ for 6 hours [12]. Further, in patients with AKI, the requirement for renal replacement therapy (RRT) was evaluated.

Categorical data were presented as numbers and percentages and were compared using either the chisquare or Fisher's exact test, when appropriate. Parametric continuous data were presented using means and standard deviations, while non-parametric continuous data were presented using medians and interquartile ranges. Continuous data were compared using Student's t-test and the Mann-Whitney U test 
for parametric and non-parametric data. The analyses were performed using IBM ${ }^{\circledR}$ SPSS Statistics version 24 (IBM Corporation, Armonk, New York, United States).

Based on data from previous studies [7, 8], 54 patients (27 patients in each group) were required to provide $80 \%$ power to detect a $31.9 \%$ difference in mortality with a $95 \%$ confidence interval. A two-sided P-value of $<0.05$ was considered statistically significant.

\section{Results}

One hundred seventy-six patient records were screened for eligibility (120 records in the Before group and 56 records in the After group). In the After group, 42 patients met the inclusion criteria, and the decision was made to include all those patients in the study to provide a more robust analysis. Therefore, a total of 42 patients in both treatment and control groups were evaluated in this study. The total number of included patients was approved by the institutional review board (IRB) of the University of Arizona. The reasons for exclusion in the Before group were the following: no mechanical ventilation $(n=34)$; no vasopressors used $(n=22)$; no ICU admission $(n=16)$; multiple ICU encounters $(n=4)$; and not confirmed sepsis diagnosis $(n=1)$. The reasons for exclusion in the After group were the following: vitamin $C$ regimen was not for sepsis diagnosis $(n=5)$; no sepsis diagnosis $(n=2)$; no vasopressors used $(n=1)$; no mechanical ventilation $(n=1)$; vitamin $C$ regimen ordered but not administered $(n=1)$; and a missing component of the vitamin $C$ regimen $(n=1)$.

Baseline characteristics were generally comparable between the two groups (Table 1). The mean age of patients in the Before group was 68 years (standard deviation (SD) \pm 14 ) and 65 years in the After group $(S D \pm 15)$. Male gender accounted for $52.4 \%$ of the Before group and $42.9 \%$ of the After group. The average lactic acid level was higher in the After group $(P=0.01)$. There were more cases of sepsis with an unknown source of infection in the Before group $(P=0.03)$. Some comorbidities were significantly higher in the Before group including hypertension $(P=0.03)$, chronic kidney disease $(P=0.005)$, and stroke $(P=$ 0.02). Although the average APACHE II scores between the Before and After groups was similar (28.81 versus 29.19, respectively; $P=0.84$ ), the average Sepsis-Related Organ Failure Assessment (SOFA) score was higher in the After group than in the Before group ( 9.74 versus 7.62 , respectively; $P=0.003$ ). The median time from admission to vitamin $\mathrm{C}$ regimen administration in the After group was 13.5 hours (Interquartile range (IQR), 6-21.75). 
Table 1

Baseline Characteristics of the Study Population

\begin{tabular}{|c|c|c|c|}
\hline Characteristic & $\begin{array}{l}\text { Before Group } \\
(n=42)\end{array}$ & $\begin{array}{l}\text { After Group } \\
(n=42)\end{array}$ & P Value \\
\hline Age, mean (SD), years & $68(14)$ & $65(15)$ & 0.34 \\
\hline Male gender, No. (\%) & $22(52.4)$ & $18(42.9)$ & 0.38 \\
\hline White blood cell (WBC), mean (SD), $\times 10^{9}$ & $15.7(10.83)$ & $12.6(9.45)$ & 0.17 \\
\hline Serum creatinine, mean (SD), mg/dL & $2.26(2.28)$ & $2.31(1.61)$ & 0.92 \\
\hline Lactic acid level, mean (SD), mmol/L & $3.83(4.12)$ & $6.25(4.65)$ & 0.01 \\
\hline \multicolumn{4}{|l|}{ Source of infection, No. (\%) } \\
\hline Pneumonia & $19(45.2)$ & $17(40.5)$ & 0.66 \\
\hline Urinary tract infection (UTI) & $6(14.3)$ & $11(26.2)$ & 0.17 \\
\hline Intra-abdominal infection & $5(11.9)$ & $10(23.8)$ & 0.15 \\
\hline Skin and soft tissue infection & $3(7.1)$ & $1(2.4)$ & 0.31 \\
\hline Endocarditis & $0(0.0)$ & $2(4.8)$ & 0.49 \\
\hline Osteomyelitis & $1(2.4)$ & $0(0.0)$ & 1.00 \\
\hline Meningitis & $0(0.0)$ & $1(2.4)$ & 1.00 \\
\hline Clostridioides difficile infection & $2(4.8)$ & $0(0.0)$ & 0.49 \\
\hline Unknown & $6(14.3)$ & $0(0.0)$ & 0.03 \\
\hline \multicolumn{4}{|l|}{ Comorbidities, No. (\%) } \\
\hline Obesity & $19(45.2)$ & $25(59.5)$ & 0.19 \\
\hline Diabetes mellitus (DM) & $17(40.5)$ & $19(45.2)$ & 0.66 \\
\hline Hypertension & $34(81)$ & $25(59.5)$ & 0.03 \\
\hline Chronic kidney disease & $19(45.2)$ & $7(16.7)$ & 0.005 \\
\hline Heart failure & $20(47.6)$ & $17(40.5)$ & 0.51 \\
\hline Coronary artery disease & $18(42.9)$ & $13(31)$ & 0.26 \\
\hline Atrial fibrillation & $21(50)$ & $16(38.1)$ & 0.27 \\
\hline Stroke & $14(33.3)$ & $5(11.9)$ & 0.02 \\
\hline Cancer & $7(16.7)$ & $8(19)$ & 0.78 \\
\hline Chronic obstructive pulmonary disease & $15(35.7)$ & $11(26.2)$ & 0.35 \\
\hline
\end{tabular}




\begin{tabular}{|llll|}
\hline Characteristic & $\begin{array}{l}\text { Before Group } \\
(\mathbf{n = 4 2 )}\end{array}$ & $\begin{array}{l}\text { After Group } \\
(\mathbf{n = 4 2 )}\end{array}$ & P Value \\
\hline Dyslipidemia & $23(54.8)$ & $18(42.9)$ & 0.28 \\
\hline Nicotine dependence & $24(57.1)$ & $17(40.5)$ & 0.13 \\
\hline Illicit drug use & $3(7.1)$ & $2(4.8)$ & 0.64 \\
\hline Alcohol abuse & $11(26.2)$ & $7(16.7)$ & 0.29 \\
\hline Liver cirrhosis & $4(9.5)$ & $4(9.5)$ & 1.00 \\
\hline Mortality assessment scores, mean (SD) & & & \\
\hline APACHE II & $28.81(8.38)$ & $29.19(8.93)$ & 0.84 \\
\hline SOFA & $7.62(2.99)$ & $9.74(3.36)$ & 0.003 \\
\hline Estimated in-hospital mortality (\%) & 55 & 55 & 1.00 \\
\hline
\end{tabular}

The study outcomes are presented in Table 2. For the primary endpoint, administration of high-dose vitamin $\mathrm{C}$, thiamine, and hydrocortisone was associated with higher hospital mortality than the control group ( $64.3 \%$ versus $42.9 \% ; \mathrm{P}=0.049$ ). For the secondary endpoints, compared to the control group, administration of high-dose vitamin $\mathrm{C}$, thiamine, and hydrocortisone was associated with a trend towards higher ICU mortality $(59.5 \%$ versus $42.9 \% ; P=0.07)$. ICU length of stay (three versus seven days; $P=0.53$ ) and duration of vasopressors ( 3.07 versus 3.12 days; $P=0.95$ ) were not statistically different between the two groups. Finally, for the safety endpoints, administration of high-dose vitamin C, thiamine, and hydrocortisone was associated with a higher incidence of acute kidney injury $(61.9 \%$ versus $26.2 \% ; \mathrm{P}=$ $0.001)$ and a trend towards a higher requirement for renal replacement therapy $(76.9 \%$ versus $45.5 \% ; \mathrm{P}=$ 0.06). This higher incidence of acute kidney injury was found in the After group even though more patients were in the Before group with chronic kidney disease $(P=0.005)$. 
Table 2

Primary, Secondary, and Safety Outcomes

\begin{tabular}{|llll|}
\hline Endpoints & $\begin{array}{l}\text { Before Group } \\
(\mathbf{n = 4 2 )}\end{array}$ & $\begin{array}{l}\text { After Group } \\
(\mathbf{n}=42)\end{array}$ & P Value \\
\hline Primary Endpoints & $18(42.9)$ & $27(64.3)$ & 0.049 \\
\hline Death in hospital, No. (\%) & & \\
\hline Secondary Endpoints & $18(42.9)$ & $25(59.5)$ & 0.07 \\
\hline Death in ICU, No. (\%) & $7(2.25-11)$ & $3(1-9.75)$ & 0.53 \\
\hline ICU length of stay, median (IQR), days & $3.12(3.56)$ & $3.07(2.81)$ & 0.95 \\
\hline Duration of vasopressors, mean (SD), days & & & \\
\hline Tertiary Endpoints & $11(26.2)$ & $26(61.9)$ & 0.001 \\
\hline Acute kidney injury, No. (\%) & $20(76.9)$ & 0.06 \\
\hline Requirement for renal replacement therapy, No. (\%) & $5(45.5)$ & & \\
\hline
\end{tabular}

The sub-group analyses were conducted to assess hospital mortality for patients receiving the exact vitamin $\mathrm{C}$ regimen components (intravenous vitamin C $1500 \mathrm{mg}$ every six hours; intravenous hydrocortisone $50 \mathrm{mg}$ every six hours; and intravenous thiamine $200 \mathrm{mg}$ every 12 hours), as well as for patients who completed the entire course of vitamin $\mathrm{C}$ regimen (four days). Results of the subgroup analyses can be found in Table 3 .

Table 3

Sub-group Analysis of the Primary Outcome

\begin{tabular}{|c|c|c|c|c|}
\hline \multirow[t]{2}{*}{ Variable } & \multirow[t]{2}{*}{ Value } & \multicolumn{2}{|l|}{ Hospital Mortality } & \multirow[t]{2}{*}{ P Value } \\
\hline & & Survived, No. (\%) & Died, No. (\%) & \\
\hline \multirow[t]{2}{*}{ Administration of the correct combination } & Yes & $7(33.3)$ & $14(66.7)$ & \multirow[t]{2}{*}{0.74} \\
\hline & No & $8(38.1)$ & $13(61.9)$ & \\
\hline \multirow[t]{2}{*}{ Administration of the complete regimen } & Yes & $3(42.9)$ & $4(57.1)$ & \multirow[t]{2}{*}{0.89} \\
\hline & No & $14(40)$ & $21(60)$ & \\
\hline
\end{tabular}

For the vitamin C regimen components, dosing variations included the following: vitamin C $1000 \mathrm{mg}$ every six hours; hydrocortisone $100 \mathrm{mg}$ every six hours; thiamine $250 \mathrm{mg}$ every 12 hours; and thiamine $500 \mathrm{mg}$ every eight hours. This study showed no difference in the hospital mortality between administering the exact vitamin $\mathrm{C}$ regimen components and changing the dose of some of the components $(P=0.74)$. 
The dosing duration of the vitamin $\mathrm{C}$ regimen is four days. However, not all the included patients received the regimen for the entire course, either because the patient died before completing the regimen or because the regimen was discontinued prematurely for other reasons. No difference in in-hospital mortality was found between the patients who received the complete regimen versus those who did not $(P=0.89)$.

\section{Discussion}

In this retrospective before-after study, the administration of high-dose vitamin C, thiamine, and hydrocortisone in critical care patients with sepsis or septic shock appeared to be associated with higher hospital mortality, higher incidence of acute kidney injury, and higher requirement for renal replacement therapy.

The results of this study are different from the previous studies $[7,8]$, which either showed that vitamin $\mathrm{C}$ regimen was effective in preventing progressive organ dysfunction, including acute kidney injury and in reducing hospital mortality [7]; or showed that vitamin $\mathrm{C}$ regimen did not improve hospital mortality [8]. The increased hospital mortality with vitamin $\mathrm{C}$ regimen in our study can be explained by the fact that the prevalence of comorbidities in our patient population is higher than in previous studies. Moreover, almost all the included patients in our study required mechanical ventilation, contributing to the negative outcomes.

The CITRIS-ALI trial was a randomized, double-blind, placebo-controlled, multi-center trial that evaluated the efficacy of high-dose vitamin $C$ versus placebo in patients with sepsis and acute respiratory distress syndrome (ARDS) [13]. All-cause mortality to day 28 was evaluated among the secondary outcomes. Mortality was $46.3 \%$ in the placebo group versus $29.8 \%$ in the vitamin $C$ group $(P=0.03)$. This trial showed the isolated effect of vitamin $\mathrm{C}$ without the combined impact of thiamine and hydrocortisone, suggesting that the negative outcome of our study might be generated from factors other than the vitamin C itself.

Our study has shown that administration of vitamin $\mathrm{C}$ regimen was associated with shorter, albeit nonsignificant, duration of vasopressor requirement $(P=0.95)$. These results are supported by The Vitamin C, Hydrocortisone, and Thiamine in Patients with Septic Shock (VITAMINS) trial, a multi-center, open-label, parallel-group randomized trial. There was no significant difference in time alive and free of vasopressors up to day seven (median of all paired differences between groups, - 0.6 hours [95\% confidence interval $(\mathrm{Cl}),-8.3$ to 7.2 hours]; $P=0.83$ ) [14]. It is worth noting that the VITAMINS trial provided the intervention for a more extended period (up to 10 days) than our study (up to four days). However, our study has shown that completing the vitamin $\mathrm{C}$ regimen did not result in a different outcome.

The safety of using vitamin $\mathrm{C}$ has been evaluated in a previous randomized, double-blind, placebocontrolled, phase I trial [15]. High-dose administration of vitamin $\mathrm{C}$ reduced the extent of multiple organ 
failure and attenuated circulating injury biomarker levels. No patients were withdrawn due to studyrelated adverse events, including hypotension, tachycardia, hypernatremia, and nausea or vomiting.

Oxalate nephropathy or nephrolithiasis has been listed as a warning in the vitamin $\mathrm{C}$ prescribing information [16]. The current study results showed a significantly higher incidence of acute kidney injury (AKI) in the vitamin C regimen group. It is worth noting that 32 patients $(76.2 \%)$ in the After group had acute kidney injury on admission, which was significantly higher than the Before group (22 patients $(52.4 \%) ; P=0.04)$. Nevertheless, the association between $A K I$ and vitamin $C$ was determined if the patient's kidney function returned to baseline after administering intravenous fluids and started to deteriorate again after initiating the vitamin $\mathrm{C}$ regimen.

The efficacy of high-dose vitamin C, thiamine, and hydrocortisone is further being investigated by The Vitamin C, Thiamine, and Steroids in Sepsis (VICTAS) trial, an ongoing prospective, multi-center, doubleblind, randomized, placebo-controlled trial (ClinicalTrials.gov Identifier: NCT03509350) [17]. The trial results could help answer some of the questions generated by the current study.

This study has several limitations. First, the study design features such as the small sample size and the retrospective before-after design may limit the generalizability of the study findings. Second, although the baseline characteristics for the included patients were primarily similar, the SOFA score in the After group was significantly higher than the Before group, which can be a contributing factor in the mortality outcome. Third, even though we used ICD-10 coding to standardize the diagnosis of sepsis, the data collection period that extended to 2015 could be a contributing factor to include patients whose diagnosis of sepsis was not according to the Third International Consensus Definitions for Sepsis and Septic Shock (Sepsis-3) [1, 2]. Fourth, a total of 35 (83.3\%) out of the 42 patients in the "after" group did not receive the complete regimen, and only 21 patients (50\%) of the 42 patients in the "after" group received the correct combination, which introduces a high degree of variability. Fifth, positive blood cultures (bacteremia) would have helped interpret the results and contribute to the differences in outcomes between the two groups. However, this data point was not available to report. Finally, the critical care service at our hospital was operated by two different providers' groups during the data collection period (i.e., a pulmonologists group from 2015 to 2017 and an intensivists group from 2017 to 2019). This difference in practice could generate a difficult variable to control or measure, and its impact on the study results cannot be ignored.

\section{Conclusions}

Our study has shown that administration of high-dose vitamin C, thiamine, and hydrocortisone in critical care patients with sepsis or septic shock within our institution appeared to be associated with harmful outcomes, including higher hospital mortality and higher incidence of acute kidney injury based on the compare groups available. However, considering the retrospective design of this study, this outcome will need to be further investigated by a randomized clinical trial. 


\section{Declarations}

\section{Funding}

This research did not receive any specific grant from funding agencies in the public, commercial, or notfor-profit sectors.

\section{Conflicts of Interest/Competing Interests}

The authors report no potential conflict of interest.

\section{Ethics Approval}

The study was approved by the institutional review board (IRB) of the University of Arizona College of Pharmacy under the expedited approval process. It was deemed to have no more than minimal risk.

\section{Consent to Participate}

The study was granted a Waiver of protected health information (PHI) Authorization (45 United States Code of Federal Regulations (CFR) 164.512(i)(2)(ii)) based on the status of IRB approval mentioned above.

\section{Consent for publication}

Not applicable.

\section{Availability of data and material}

All study data and materials comply with the health science research standards and followed The University of Arizona Health Sciences policies and procedures.

\section{Code availability}

Not applicable. 


\section{Authors' contributions}

HAW had full access to all the data in the study and took responsibility for the integrity of the data and the accuracy of the data analysis, including and especially any adverse effects. HAW, WM, and JM contributed substantially to the study design, data analysis and interpretation, and manuscript writing.

\section{References}

1. Seymour CW, Liu VX, Iwashyna TJ, et al. Assessment of clinical criteria for sepsis: for the third international consensus definitions for sepsis and septic shock (sepsis-3). JAMA. 2016;315(8):76274.

2. Shankar-Hari M, Phillips GS, Levy ML, et al. Developing a new definition and assessing new clinical criteria for septic shock: for the third international consensus definitions for sepsis and septic shock (sepsis-3). JAMA. 2016;315(8):775-87.

3. Sepsis data and report. Centers for disease control and prevention. Available at https://www.cdc.gov/sepsis/datareports/index.html. Accessed on August 30, 2019.

4. Paoli CJ, Reynolds MA, Sinha M, et al. Epidemiology and costs of sepsis in the United States-an analysis based on timing of diagnosis and severity level. Crit Care Med. 2018;46(12):1889-97.

5. Rhodes A, Evans LE, Alhazzani W, et al. Surviving sepsis campaign: international guidelines for management of sepsis and septic shock: 2016. Crit Care Med. 2017;45(3):486-552.

6. Wilson JX. Mechanism of action of vitamin C in sepsis: ascorbate modulates redox signaling in endothelium. Biofactors. 2009;35(1):5-13.

7. Marik PE, Khangoora V, Rivera R, Hooper MH, Catravas J. Hydrocortisone, vitamin C, and thiamine for the treatment of severe sepsis and septic shock: a retrospective before-after study. Chest. 2017;151(6):1229-38.

8. Litwak JJ, Cho N, Nguyen HB, et al. Vitamin C, hydrocortisone, and thiamine for the treatment of severe sepsis and septic shock: a retrospective analysis of real-world application. J Clin Med. 2019;8(4):478.

9. Ahn JH, Oh DK, Huh JW, et al. Vitamin C alone does not improve treatment outcomes in mechanically ventilated patients with severe sepsis or septic shock: a retrospective cohort study. J Thorac Dis. 2019;11(4):1562-70.

10. Harris PA, Taylor R, Thielke R, et al. Research electronic data capture (REDCap) - a metadata-driven methodology and workflow process for providing translational research informatics support. $\mathrm{J}$ Biomed Inform. 2009 Apr;42(2):377-81. 
11. Harris PA, Taylor R, Minor BL, et al. The REDCap consortium: Building an international community of software platform partners. J Biomed Inform. 2019;95:103208.

12. KDIGO clinical practice guideline for acute kidney injury. Section 2: AKI definition. Kidney Int Suppl. (2011). 2012;2(1):19-36.

13. Fowler AA, Truwit JD, Hite RD, et al. Effect of vitamin C infusion on organ failure and biomarkers of inflammation and vascular injury in patients with sepsis and severe acute respiratory failure: the CITRIS-ALI randomized clinical trial. JAMA. 2019;322(13):1261-70.

14. Fujii T, Luethi N, Young PJ, et al. effect of vitamin C, hydrocortisone, and thiamine vs hydrocortisone alone on time alive and free of vasopressor support among patients with septic shock: the VITAMINS randomized clinical trial. JAMA. 2020;323(5):423-31.

15. Fowler AA, Syed AA, Knowlson S, et al. Phase I safety trial of intravenous ascorbic acid in patients with severe sepsis. J Transl Med. 2014;12:32.

16. Ascor (ascorbic acid). [prescribing information]. Santa Ana: McGuff Pharmaceuticals, Inc; October 2017.

17. Hager DN, Hooper MH, Bernard GR, et al. The vitamin C, thiamine, and steroids in sepsis (VICTAS) protocol: a prospective, multi-center, double-blind, adaptive sample size, randomized, placebocontrolled, clinical trial. Trials. 2019;20(1):197. 